\title{
ABSTR ACTS
}

\section{EAR}

Healing of Experimental Labyrinthine Fistulas: Further Observations. J. R. LinDSAY, M.D. (Chicago). Arch. Otolaryng., I947, xlvi, 5, 584-60o.

Further observations on factors influencing osteogenesis at the site of the labyrinthine fistula are presented and the rôle of the covering flap is considered in particular.

The interpretation based on the previous experiments that success in maintaining a patent fenestra in a semi-circular canal is dependent on the degree to which the membranous canal is preserved in its normal position at the outer periphery of the bony canal, where it is free to come into direct contact with whatever covering may be used over the fistula, appears to be supported.

R. B. LUMSDEN.

\section{Symposium on Fenestration of the Labyrinth}

Lempert Fenestra Nov-Ovalis Operation for the Restoration of serviceable unaided hearing in Patients with Clinical Otosclerosis : Its present evolutionary status. Julius Lempert, M.D. (New York).

Osteogenesis following Fenestration of the Vestibular Labyrinth of the. Rhesus Monkey: A controlled experimental study. JULIUS LEMPERT, M.D. (New York) ; Philip E. Meltzer, M.D. (Boston) ; Le Roy A. Schall, M.D. (Boston) ; DoRothy WolfF, Ph.D. (New York).

Revision of the Fenestration Operation. PHILIP E. Meltzer, M.D. (Boston). Medical and Surgical care of the patient selected for Fenestration of the Labyrinth. K. M. DAY, M.D. (Pittsburgh).

Training of Surgeon and selection of patient for the Fenestration Operation. J. H. Maxwell, M.D., Ann Arbor, Mich.

General Correlation. Marvin F. Jones, M.D. (New York). Arch Otolaryng., I947, xlvi, 4, 478-548.

In his article entitled "General Correlation", Marvin F. Jones summarizes the lessons to be learned from this symposium as follows :

I. "There is a definite and too great element of error in the present methods of arriving at a diagnosis which precludes dogmatism.

2. "Training methods for the development of understanding, capable fenestration surgeons should be subject to the approval of authoritative bodies.

3. "Certification of those trained in fenestration surgery should be implemented by the American Board of Otolaryngology at once as a protection to the public.

4. "Anæsthesia for fenestration should receive more attention on the basis of blood control and safety. 


\begin{abstract}
s
5. "The Lempert endaural fenestration operation performed by a competent otologic surgeon, properly trained, improves hearing to the useful level. The patient's hearing can be maintained for ten years in an indefinite percentage of selected cases. The figures reported thus far are so divergent that it seems inadvisable to state a definite percentage. I would feel justified in saying there is a permanent improvement in more than $5^{\circ}$ per cent.

6. "A comment which I deem in order concerns the medical information liberated to the lay press. The public is entitled to know the applicable advances in medicine. Unfortunately, there has been no distinction between reporting experimental progress and reporting established procedures. The first enthusiastic statements are not apt to be the final, soberly considered deductions. The old pendulum idea holds also in medicine. The first flush of optimistic endeavour is sometimes followed by an overly pessimistic phase as the other extreme. Opinion will finally settle down to correct values."
\end{abstract}

R. B. LumsDen.

Symposium on Vertigo before The American Otological Society (Ann. Otol., Rhin. and Laryng., I947, lvi, 5I4.)

The Symposium takes the form of four papers followed by a Summation.

I. The Physiology of the Vestibular Mechanism in Relation to Vertigo. W. J. McNally (Montreal), 5I4.

"Vertigo may be defined as a consciousness of discord in the postaural mechanism. Maintenance of posture depends upon information received, the labyrinths . . . (and other) so-called Kinesthetic sensations. This information is correlated in the consciousness, and conflicting information from any source, or failure of the central nervous system to co-ordinate this information leads to confusion in the mind of the individual; this is what we call vertigo or dizziness."

Following the above definition of vertigo, the study is divided into sections. The first is a concise review of the experimental work on the subject during the last hundred years, well worthy of reference, but so concise as to defy further abstraction. The second part is devoted to vestibular tests.

The Slow Tilt Test. Grahe describes two. In the first the patient, strapped to a frame is tilted from an oblique to the vertical position with his eyes closed. Failure to detect the vertical position is indicative of utricular dysfunction. In the second test, the patient is tilted about a horizontal axis. Normally the head should maintain the erect position; failure to do so again indicates utricular dysfunction. These tests have not received general acceptance.

The Rapid Tilt Test. The reaction of a patient to rapid tilting is primarily a test of vertical semi-circular canal function. It has the objection that, in a lesion of long standing compensation may develop and the reaction appear normal.

Caloric Stimulation of the Labyrinth. This test has the advantage over the rotation test that it stimulates only one labyrinth at a time, and over the galvanic in that it stimulates the end organ only and not the nerve. Bárány's Convection current theory is probably the best explanation of the reaction. 


\section{Ear}

Kobrak's method of minimal stimulation is generally regarded as the best method of application. While it is known that the reaction may vary in the same subject from day to day, there is no evidence of habituation and lessening sensitivity with repeated stimulation. Recent experiments have cast some doubt on the validity of Ewald's Law of maximal and minimal stimulus.

It is stressed that " a clinical vestibular test must be as all informative as possible and yet it should be as simple as possible."

At the present time the vestibular tests in common use rely on the reaction of the eye muscles to vestibular stimulation. In point of fact the whole musculature of the body is under the influence of the labyrinth. It is probable that the tests will be more revealing when we have a greater knowledge of the response in other muscle groups.

\section{The Symptomatology of Vertigo. Alfred LEWY (Chicago), 534.}

Vertigo like headache may occur as a symptom of almost any ill to which the flesh is heir. The point of origin may lie in the field of internal medicine otology, neurology or ophthalmology, but the symptom becomes manifest through disturbance or destruction of the vestibular centres of the brain.

Vertigo of peripheral origin is recognized by the associated aural manifestations, local objective lesion, deafness and tinnitus, and, if present, a falling reaction influenced by head position.

Many of the cases of vertigo not demonstrably due to local peripheral disorder are of toxic origin :-focal infection, fumes (e.g. Carbon Monoxide) or drugs being administered for some other condition, a recent example being streptomycin. Diagnosis is by recognition of the potential cause and its elimination.

The majority of central causes of vertigo, affect other nerves than the VIIIth, and to a large extent their diagnosis depends on recognition of these associated findings.

Postaural nystagmus is, in the writer's opinion, invariably of central, generally subtentorial, origin, as also are all forms of vertical nystagmus. A coarse, persistent, slow nystagmus of vestibular type not influenced by head position, and increasing instead of decreasing with time, suggests a cerebellar lesion, certainly if it is toward a dead labyrinth. A diminished or lost labyrinthine response may be due to either a central or a peripheral lesion, but a perverted response invariably denotes a central lesion.

The paper concludes with Leidler's classification of vestibular symptoms (vide Leidler, R. Pract. Otol., Rhin. Laryng., May and June, I939, ii, 86 and I52).

(i) Vestibular symptoms suggestive of central nervous system disease.

I. Severe attacks of dizziness over a long period with constant hearing.

2. Unilateral or bilateral disturbance of vestibular reaction with constant normal hearing.

3. Long lasting spontaneous nystagmus of at least second degree with normal hearing.

4. Occurrence of severe headache or increase of existing headache simultaneous with severe dizziness.

5. Perverted nystagmus reactions, especially predominance of the 


\section{Abstracts}

slow component and disturbances of rhythm (in the direction of conjugate deviation).

6. Postural nystagmus.

7. High grade nystagmus without dizziness, or if both are present independent of each other.

(ii) Vestibular signs conclusive of central nervous system disease.

I. Unilateral loss of hearing and vestibular response with spontaneous nystagmus of at least second degree, to the same side.

2. Unchanged second degree nystagmus in the same direction for longer than a week.

3. Continued second degree pure rotary nystagmus over a long period.

4. All forms of vertical nystagmus.

5. Unilateral or bilateral caloric inexcitability in the presence of normal hearing and normal rotation response.

6. One phase reactions (conjugate deviation).

7. Dizzy spells with loss of consciousness.

8. Occurrence of long lasting changes of head posture during examination of the vestibular nerve, sometimes with somnolence or sleep.

\section{Pathology of Vertigo arising from the Peripheral Vestibular Apparatus.}

J. R. Lindsay (Chicago), 54I.

I. The pathologic disturbances which are associated with most clinical conditions giving origin to pseudo-Ménière's syndrome (vertigo without auditory symptoms or central neurologic signs) are not known. The localization has rarely been proven. The postural vertigo which is a characteristic of this group usually corresponds to the type which is most frequent in proven disease of the posterior fossa in that a positional nystagmus occurs which changes direction with alteration of the position of the head. A central origin is therefore suggested.

2. Vertigo of peripheral origin has been satisfactorily explained in labyrinthitis of various types, tumors and fractures of the labyrinth.

3. In concussion of the labyrinth the pathologic lesion has not been adequately demontrated. A temporary threshold dip at the $\mathrm{C}$ frequency has been shown. A subarachnoid hæmorrhage which extends along the vessels and nerves into the porus acusticus and even into the perilymphatic spaces is common after head trauma and following craniotomy.

The degree of hæmorrhage into the porus acusticus and the perilymphatic space which is necessary to interfere with function is, however, indefinite. Probably a frank hæmorrhage into the labyrinthine spaces is necessary for any marked disturbance of function.

The pathologic explanation for the apoplectic onset of deafness, tinnitus and vertigo in a previously healthy ear has not been demonstrated. An interruption of circulation or a hæmorrhage are considered to be most probable.

4. The pathogenesis of a toxic neuritis or neurolabyrinthitis is not clear in all cases. For example, the occurrence of progressive neural degeneration in otosclerosis, as well as that which sometimes occurs as a complication after the fenestration operation, has created special interest.

5. The histopathologic disturbance in idiopathic labyrinthine dropsy lacks an ætiologic explanation. 


\section{Ear}

Degeneration of neural elements appears to be a late accompaniment of this type of hydrops, whereas in serous labyrinthitis the degeneration of neural elements occurs early.

Degeneration of sensory structures within the endolymphatic spaces and the stria vascularis appears to parallel the development of hydrops in serous labyrinthitis but may be slight or absent in comparatively long standing cases of idiopathic hydrops. (Author's Summary.)

IV. Incidence of Vertigo in Neurological Conditions. I. LEVY and J. L. O'Leary (St. Louis), 557.

The neurological evaluation of vertigo as a symptom is dependent to a large extent on the associated phenomena. The author discusses the various locations of lesions which may give rise to vertigo. In general terms lesions of acute onset are more liable to produce this symptom than the more insidiously developing lesions. Vertigo has not been a particularly useful symptom in contributing to the diagnosis of cerebellar lesions.

Eight cases are presented in detail, illustrating different problems in the differential diagnosis of Ménière's Disease.

V. Summation. A. C. Furstenberg. Ann Arbor, 576 .

I. Vertigo may be a symptom of some toxic disorder. Its occurrence in septic patients, after the administration of certain drugs and more recently of the antibiotic streptomycin, is considerable proof of this assumption.

2. Vertigo may be one of the manifestations of a nervous depression. The diagnosis is usually not difficult, although a satisfactory therapeutic response is not always obtainable. In this connection a word of caution is offered. The otologist must be careful not to do or say something that will fix the patient's symptoms upon some specific cause. When one offers a precise explanation for the patient's ills and assures successful results from treatment or an operation there is always the risk of a therapeutic failure and the permanent crystallization of the patient's symptoms. The sedative dilantin has seemed to benefit some of these patients.

3. Vertigo is occasionally a symptom of hypertensive disease. The prospect of relieving the patient of this troublesome condition is favourable if surgical measures result in a substantial reduction in blood pressure.

4. Organic lesions of the central nervous system may produce vertigo but they are usually associated conspicuously with other clinical signs of intracranial disease. The classical example is the cerebellopontine angle tum sur.

5. There are instances when vertigo is the result of an end organ lesion in the acoustic nerve. The vertigo derived from this source, as for example that of Ménière's disease, is so specifically characteristic as not to be confused with any other type. I speak succinctly and without fear of derision that if the patient suffers a violent paroxysmal vertiginous attack-one that fells him in his tracks-we are dealing in all probability with an end organ lesion, not an intracranial disease. (Author's summary.)

Note. The above papers are so condensed that it is impossible to abstract them in any detail. Those interested in the subject are recommended to read them in their original.

E. J. Gilroy Glass. 


\section{Abstracts}

\section{ESOPHAGUS}

Treatment of Esophageal Varices by Injection of a Sclerosing Solution. Herman J. Moersch, M.D. (Rochester, Minn.). Jour. A.M.A., November 22nd, I947, cxxxv, 12, 754 .

Roentgenological examination is of great value in the diagnosis of œsophageal varices but is not infallible. In doubtful cases œsophagoscopy should be employed.

The writer reports results of treatment in twenty-two cases of cesophageal varices in which gastro-intestinal hæmorrhage occurred. The patients were treated by the injection of sodium morrhuate into the varices through an csophagoscope. All patients were followed for a period of at least three years. Twelve of the patients have had no further bleeding after treatment. Patients in whom unsatisfactory results were obtained, invariably were found to have varices in the cardiac end of the stomach as well as in the oesophagus.

Where the stomach is involved as well as the osophagus, some other form of treatment such as portal caval anastomosis or the resection of the cardiac end of the stomach and the lower end of the osophagus should be considered.

The article is illustrated, has three tables and a bibliography.

\section{Angus A. Campbell.}

\section{MOUTH}

Diseases of the Salivary Glands. A. C. Furstenberg, M.D. (Ann Arbor, Michigan). Jour. A.M.A., January 3rd, I948, cxxxvi, I, I.

This discussion is limited to a consideration of parotid and submaxillary glands. The commonest cause of infection in these glands is due to the presence of a salivary calculus and its most frequent location is in Wharton's duct, although it sometimes occurs in Stensen's duct. When the gland is acutely inflamed, antibiotics and symptomatic treatment should be employed. During the quiescent period, the stone should be removed surgically by the intra-oral route. When the glands are chronically inflamed, it may be necessary to remove the whole gland by external operation.

Syphilis, actinomycosis and tuberculosis are rarely found. Injury and dehydration are potent factors in these infections. Pilocarpine therapy is frequently effective in inflammatory cases, accompanied by dehydration.

The commonest type of neoplasm is the mixed tumour. In its removal, the surgeon must not fail to eradicate the lesion completely for when it recurs, it usually presents carcinomatous changes and further surgical efforts are futile.

Angus A. Campbell.

\section{MISCELLANEOUS}

Scleroma: A Clinico-Pathological Study of Seven Cases in one Family. H. James Hara, Orlyn B. Pratt, Milton G. Levine and Robert E. Hoyt (Los Angeles, California). Ann. Otol., Rhin. Laryng., September, I947, lvi, 769 .

I. A detailed study on seven cases of scleroma from one family is presented.

2. Histologic changes in the mucous membrane of the respiratory tractfrom the incipient nasal catarrh, followed successively by suppurative rhinitis, atrophic rhinitis, chronic granuloma to the final deforming scleromatous scarare shown. 


\section{Miscellaneous}

3. Of the various therapeutic measures tried streptomycin in an adequate dose is the most promising.

4. A diagnosis of scleroma can be made by trained bacteriologists and serologists long before histologic changes are recognized. (Author's Summary.)

Bell's Palsy. Pathology and Surgery. A Report concerning fifty patients who were operated on after the method of Ballance and Duel. KARSTEN KetTel, M.D. (Hillerod, Denmark). Arch. Otolaryng., 1947, xlvi, $427-472$.

The author sets out to estimate the value of the operation and to try to arrive at a deeper understanding of the pathology.

Changes which have not been described earlier have been demonstrated both in the mastoid cells, and in the facial canal itself.

\section{Etiology and Pathogenesis}

Much seems to indicate that Bell's palsy is a pathogenetic entity, the primary and central feature of which is a "dysregulation" of the circulation, which probably takes place near the stylomastoid foramen. In most cases the nerve as the most susceptible tissue suffers alone ; in other cases the surrounding, more resistant bone is also affected. The result is ischæmic paralysis and ischæmic bony necrosis, respectively. The consequence of the lack of blood supply of the nerve is œdema with subsequent degenerative changes; the nerve is thus compressed in its bony canal, which causes further impairment of the vascular supply, so that a vicious circle arises, the process being reversible. Thus the actual cause of the paralysis is the ischæmia ; the compression in the fallopian canal is only a secondary phenomenon, and not, as maintained by most authors, the real cause of the paralysis. In extreme cases ischæmic necrosis of the nerve may be the result.

\section{Surgical Therapy}

In 85 to 90 per cent. of the cases Bell's paresis subsides under suitable conservative treatment. In selecting the the last Io to I5 per cent. of the patients, Anglo-American authors rely on the faradic reaction, maintaining that if the response becomes negative the patient will not recover at all or will only recover partly if conservative treatment is instituted. It is shown that this does not hold true, and the explanation is that the faradic stimulus is of too short duration to excite contractions of the musculature, the chronaxia of which has become extended.

Fifty cases have been treated surgically on the basis of the following three indications :-

I. A decompression should be done in cases in which signs of beginning mobility have not appeared after an observation period of two months.

Indication ( $I$ ) ought to be considered as serving temporarily until better means have been obtained for selecting the early cases for operation.

2. A decompression should be done if the spontaneous recovery of mobility has ceased before complete restitution has been obtained.

3. Decompression is indicated in relapsing paresis.

R. B. Lumsden. 


\begin{abstract}
s
The Antihistaminic Drugs. George L. Waldbott, M.D. (Detroit). Jour. A.M.A., September 27th, I947, cxxxv, 4, 207.

The most promising of these drugs are antistin, neoantergan, pyribenzamine hydrochloride and benadryl hydrochloride. They inhibit secretion induced by histamine in the lacrimal and salivary glands and the mucous glands of the bronchial tree. They produce local anæsthesia when injected as well as when applied locally. They do not neutralize histamine chemically nor do they prevent its production in the body. They are believed to compete with histamine in its affinity for the cells. They are entirely palliative, since symptoms recur in four to six hours. There is no indication of a cumulative action or of addiction to the drug. In hay fever, they are most beneficial during the first part of the season when the nasal mucosa secretes clear, watery fluid and when there is no evidence of secondary infection. Waterlogged mucous membranes and nasal polyps shrink visibly within thirty minutes after ingestion of the drug. This is also true in perennial allergic rhinitis in the absence of suppurative changes.

Side effects, such as drowsiness, dizziness, headaches, nausea, dryness in the throat and muscular twitching may appear with the first dose and be absent later or vice versa.

The article has three figures, two tables and a bibliography.
\end{abstract}

ANgus A. CAMpbell. 